\title{
Synthesis, Characterization and Third Order Non Linear Optical Properties of Metallo Organic Chromophores
}

\author{
Nallamuthu Ananthi ${ }^{1}$, Umesh Balakrishnan ${ }^{1}$, Sivan Velmathi ${ }^{1 *}$, \\ Krishna Balakrishna Manjunath ${ }^{2}$, Govindarao Umesh ${ }^{2}$ \\ ${ }^{1}$ Department of Chemistry, National Institute of Technology, Tiruchirappalli, India \\ ${ }^{2}$ Department of Physics, National Institute of Technology, Surathkal, India \\ Email: *velmathis@nitt.edu
}

Received December 17, 2011; revised January 16, 2012; accepted January 26, 2012

\begin{abstract}
The organic imine and their metal complexes were synthesized and characterized by IR, UV and NMR. The third order non linear optical properties of the compounds were investigated. The measurements of second hyperpolarizabilites were performed using single beam Z-scan technique with 8 ns laser pulses. Ligand and its Copper, Zinc and Nickel complexes show good third order non linearity whereas Manganese complex did not show any activity.
\end{abstract}

Keywords: Third Order NLO Properties; Salen Ligand; Transition Metal Complexes; Z-Scan Technique

\section{Introduction}

The non linear optical properties of the $\pi$ conjugated organic materials have been widely investigated due to their possible applications in a variety of optoelectronic and photonic applications [1-3]. In third order non linear optics, guidelines for the optimization of the second hyperpolarizability $\gamma$ of any organic molecule have been steadily improving but the understanding is far less developed than for the first hyperpolarizability $\beta[4,5]$. So it is desirable to optimize the $\gamma$ values. In particular the strong delocalization of $\pi$ electrons or the presence of any hetero atoms in the organic backbone determines a very high molecular polarizability and thus remarkable third order optical nonlinearity. In general large hyperpolarizabilities are the result of an optimum combination of various factors such as $\pi$ delocalization length, donor-acceptor groups, dimensionality, conformation and orientation for a given molecular structure [6,7].

Organic materials with high nonlinear optical (NLO) susceptibilities are considered to be potential alternatives to the inorganic materials for applications in optoelectronics [8-10]. Reports of devices, such as intra cavity frequency doublers based on the second-order NLO effect in an organic crystal, have already started to appear in the literature. As a result, there is an increasing interest in designing new organic materials with desired linear and nonlinear optical properties.

Crystal structure, spectroscopic studies, and nonlinear optical (NLO) properties of Schiff base metal complexes containing an N, S donor ligand have been studied by

\footnotetext{
"Corresponding author.
}

Zhao-Ming Xue et al. [11]. Third-order nonlinear optical properties of nickel(II) and copper(II) complexes with salen ligands, functionalized with electron donor/acceptor groups (DA-salen), have been investigated in solution by the Z-scan technique using an Nd:YAG laser (1064 $\mathrm{nm}) \cdot[12]\left[\mathrm{Ag}(\mathrm{L})_{2}\right]\left(\mathrm{NO}_{3}\right) \cdot(\mathrm{MeOH}) \cdot(\mathrm{EtOH})$ and $\left[\mathrm{HgI}_{2}(\mathrm{~L})\right]$ $\{\mathrm{L}=1,2$-bis [(ferrocen-l-yl methylene) amino] ethane $\}$ have been prepared, structurally characterized and thirdorder nonlinear optical properties have been studied. The results indicate that the two complexes exhibit very strong NLO absorption and strong self-focusing effects [13]. The third-order susceptibilities $\chi^{3}$ of octa substituted metallophthalocyanines have been measured by third-harmonic generation experiments. The effect has been associated to the role of two-photon allowed metal possibly $(d-d)$ transitions in the cobalt derivative [14]. A novel zinc (II) 1,3,5 -triazine-based complex [ $\left.\mathrm{Zn}(\mathrm{TIPT}) \mathrm{Cl}_{2}\right] \cdot 2 \mathrm{CH}_{3} \mathrm{OH}(\mathrm{TIPT}=$ 2,4,6-tri(2-ispropylidene-1-ly)hydrazono-1,3,5-triazine) was prepared and structurally characterized and the hyperpolarizability $\gamma$ value was found to be $8.26 \times 10^{-30}$ esu [15].

In this paper we worked out the third order non linear optical parameters of the synthesized salen imine and its metal $(\mathrm{Cu}, \mathrm{Zn}, \mathrm{Ni}, \mathrm{Mn})$ complexes using $\mathrm{Z}$-scan measurement using $7 \mathrm{~ns}$ laser pulses at $532 \mathrm{~nm}$. Herein we report, the effects of the chromophore hydroxyl group on this imine and also the role of different metals on the non linear optical property. The structures of the compounds studied are represented in Scheme 1.

\section{Experimental Section}

2,4-dihydroxy benzaldehyde, o-amino phenol were pur- 


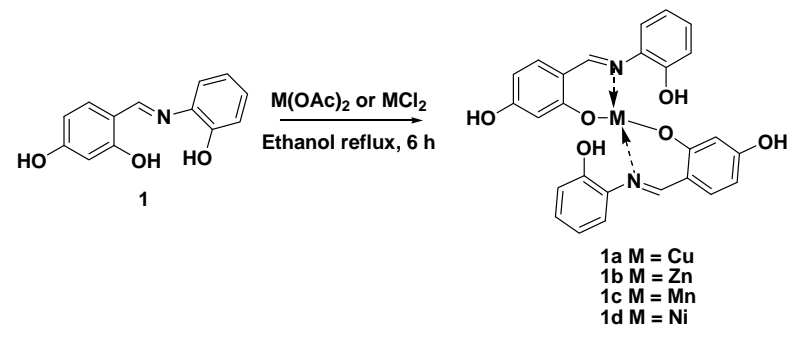

Scheme 1. Synthesis of Schiff base metal complexes.

chased from Aldrich chemicals and copper, zinc, manganese acetates and the nickel chloride were purchased from Loba chemie and used as received. Ethanol, Dimethy formamide (DMF) and other solvents were purified by the reported procedure.

\subsection{Synthesis of Schiff Base 1}

To the refluxing ethanolic solution of o-amino phenol (5 mmol, $0.546 \mathrm{~g}$ ), 2,4-dihydroxy benzaldehyde $(5 \mathrm{mmol}$, $0.4755 \mathrm{~g}$ ) in ethanol was added by drops and then reflux in a water bath for three hours. Then the imine was separated by hexane wash in $80 \%$ yield.

Melting point $\left({ }^{\circ} \mathrm{C}\right): 78$ - 80, IR data: $v\left(\mathrm{~cm}^{-1}\right) 3312$ (-NH), 3390, $1056(-\mathrm{OH}), 1741\left(-\mathrm{COOCH}_{3}\right), 1644(\mathrm{C}=\mathrm{N})$, ${ }^{1} \mathrm{H}$ NMR data $\left(\mathrm{CDCl}_{3}, \mathrm{TMS}, \delta\right.$, ppm): $6.4(\mathrm{~d}, 1 \mathrm{H},-\mathrm{CH})$, $6.7(\mathrm{~m}, 2 \mathrm{H},-\mathrm{CH}), 7.12(\mathrm{~m}, 2 \mathrm{H},-\mathrm{CH}), 7.54(\mathrm{~s}, 1 \mathrm{H},-\mathrm{CH})$, $7.78(\mathrm{~d}, 1 \mathrm{H},-\mathrm{CH}), 8.87(\mathrm{~s}, 1 \mathrm{H},-\mathrm{HC}=\mathrm{N}), 9.85(\mathrm{~s}, 3 \mathrm{H}$, -OH). ${ }^{13} \mathrm{C}$ NMR $\left(\mathrm{CDCl}_{3}\right.$, TMS, $\delta$, ppm): 103.7, 108.6, $110.9,117.2,122.5,123.6,128.7,132.4,141.2,151.5$, $159.8,162.3,162.5$.

\subsection{Synthesis of Metal Complexes 1a, 1b, 1c, 1d from Schiff Base 1}

To the stirred solution of the imine $(1 \mathrm{mmol})$, the metal starting compounds $\mathrm{Cu}\left(\mathrm{CH}_{3} \mathrm{COO}\right)_{2} \cdot \mathrm{H}_{2} \mathrm{O}$,

$\left.\mathrm{Zn}\left(\mathrm{CH}_{3} \mathrm{COO}\right)_{2} \cdot 2 \mathrm{H}_{2} \mathrm{O}, \mathrm{Mn}\left(\mathrm{CH}_{3} \mathrm{COO}\right)_{2} \cdot 2 \mathrm{H}_{2} \mathrm{O}\right)$ and

$\mathrm{NiCl}_{2} \cdot 6 \mathrm{H}_{2} \mathrm{O}(1 \mathrm{mmol})$ was added in an ethanolic solution. Then the reaction mixture was reflux for six hours. Then the metal complexes were separated by washing with dichloromethane to remove the unreacted imine. Yield: $75 \%$.

1a: Melting point $\left({ }^{\circ} \mathrm{C}\right):>360$. IR data: $v\left(\mathrm{~cm}^{-1}\right): 3430$, $1038(-\mathrm{OH}), 1622(\mathrm{C}=\mathrm{N})$.

1b: Melting point $\left({ }^{\circ} \mathrm{C}\right):>360$. IR data: $v\left(\mathrm{~cm}^{-1}\right): 3433$, $1035(-\mathrm{OH}), 1620(\mathrm{C}=\mathrm{N})$.

1c: Melting point $\left({ }^{\circ} \mathrm{C}\right):>360$. IR data: $v\left(\mathrm{~cm}^{-1}\right): 3437$, $1039(-\mathrm{OH}), 1616(\mathrm{C}=\mathrm{N})$.

1d: Melting point $\left({ }^{\circ} \mathrm{C}\right):>360$. IR data: $v\left(\mathrm{~cm}^{-1}\right): 3436$, $1037(-\mathrm{OH}), 1624(\mathrm{C}=\mathrm{N})$.

\subsection{Transmission Measurements}

The transmission coefficient of the samples synthesized was measured by Z-scan technique using Q-switched laser pulses at a wavelength of $532 \mathrm{~nm}$. Z-scan technique introduced by Sheik-Bahae et al. [16] is an extensively utilized experimental tool for studying optical nonlinearities in a wide class of materials. The technique relies on the fact that the light intensity varies along the axis of a convex lens and is maximum at the focus. By moving the sample through the focus, the intensity dependent absorption is measured as a change of the transmission through the sample. This technique is an increasingly popular method for the measurement of the non linear absorption coefficient $(\beta)$ and the non linear refractive index $\left(\eta_{2}\right)$ of the samples and has the advantages that it immediately indicates the sign and type of non linearity (refraction or absorption). A frequency doubled Q-Switched Nd-YAG Laser (Spectra Physics GCR-170), which produces $8 \mathrm{~ns}$ laser pulses at $532 \mathrm{~nm}$ was used as the light source. The laser beam was focused by plano-convex lens of $25 \mathrm{~cm}$ focal length. For the measurements, liquid samples of the concentration $1 \times 10^{-3} \mathrm{~mol} /$ liter were prepared in research grade DMF. The solutions were then taken in a quartz cuvette of thickness $1 \mathrm{~mm}$ and the cuvette was mounted on a motorized linear translation stage for translating the sample across the focal spot. The beam waist at the focus was estimated to be $18.9 \mu \mathrm{m}$ and the corresponding Raleigh length was $2.11 \mathrm{~mm}$. A circular aperture, of size $5 \mathrm{~mm}$, is mounted in front of the photo detector placed at a distance of about $15 \mathrm{~cm}$ from the focal spot of the laser beam. The light intensity transmitted by the sample is measured as a function of the sample position along the Z-axis (the beam axis) thereby obtaining the NLO refractive index $\left(\eta_{2}\right)$ "closed aperture" Z-scan data. The measurements were repeated after removing the aperture in order to obtain the NLO absorption index $(\beta)$ "open aperture" Z-scan data. The closed aperture data was then divided by the open aperture data to generate the normalized transmission versus translation distance data, using which the nonlinear optical susceptibility was calculated following the analysis method of Sheik-Bahae et al. [16].

\section{Results and Discussion}

\subsection{Synthesis and Characterization of Ligand and Metal Complexes}

The Schiff base 1 was synthesized by the condensation of 2,4-dihydroxy benzaldehyde with o-amino phenol. The compound was well characterized by UV-vis, FTIR, ${ }^{1} \mathrm{H}$ and ${ }^{13} \mathrm{C}$ NMR spectroscopy methods before studying their NLO properties.From the electronic absorption spectra of the ligand, it was observed that there is no absorption band in the visible region. The bands in the region $207-230 \mathrm{~nm}$ could be assigned to excitation of the $\pi$ electrons of the aromatic system. The observed bands in the range $316-340 \mathrm{~nm}$ can be attributed to the $n-\pi^{*}$ 
transition. The band in the region around $260-284 \mathrm{~nm}$ is due to the electronic transition between the $\pi$ orbital localized on the azomethine group $(\mathrm{C}=\mathrm{N})$. The band in the region $208-225 \mathrm{~nm}$ could be assigned to excitation of the $\pi$ electrons of the aromatic system. This UV-vis spectrum also indicates that there is negligible one photon absorption at a wavelength of $532 \mathrm{~nm}$ wavelength. Thus, in all the experiments, the nonlinear absorption behavior can be attributed to the two photon absorption (TPA).

In the electronic spectra of metal complexes, Nickel and copper complexes shows bands at $275 \mathrm{~nm}$ which are assigned to intra ligand electronic transitions $\left(\pi-\pi^{*}\right)$. In zinc complex red shift has taken place compared to copper and nickel complexes, the band appears at $290 \mathrm{~nm}$. This is due to the diamagnetic behavior of the $\mathrm{Zn}$ (II) ions whereas the $\mathrm{Ni}(\mathrm{II})$ and $\mathrm{Cu}$ (II) are paramagnetic. An intense peak around $420 \mathrm{~nm}$ are assigned to ligand to metal charge transfer. The bands with higher energy in the UV region are of intra-ligand $\pi-\pi^{*}$ type or charge transfer transitions involving energy levels which are higher in energy than the ligand's Lowest Unoccupied Molecular Orbital (LUMO).

In the FTIR spectroscopy we get information about the presence of functional groups in the compound such as $\mathrm{C}=\mathrm{C}, \mathrm{CH}=\mathrm{N}$ etc. by the position of peaks which arise due to stretching vibration of the bonds in the groups. The presence of $\mathrm{C}=\mathrm{N}$ in the molecule is confirmed by the vibration observed between $1690-1640 \mathrm{~cm}^{-1}$ and aromatic $\mathrm{C}=\mathrm{C}$ between $1600-1473 \mathrm{~cm}^{-1}$. In the IR spectra of metal complexes, $\mathrm{C}=\mathrm{N}$ bond stretching was slightly shifted to the lower region (1616 - $1624 \mathrm{~cm}^{-1}$ ) confirming the coordination of $\mathrm{C}=\mathrm{N}$ bond of the ligand to the metal. All the other function groups also show their stretching in the respective regions in the IR spectra.

NMR spectroscopy is one of the principal techniques which give us the structural information about molecules. NMR spectra were obtained using deuterated dimethyl sulphoxide (DMSO- $\mathrm{d}_{6}$ ) as the solvent. Compound $\mathbf{1}$ gave a singlet at around $\delta 8.8 \mathrm{ppm}$ corresponding to the $\mathrm{CH}=\mathrm{N}$ proton indicating the formation of imine and the aromatic protons resonate in the $\delta 6.4-7.7$ region. In addition, the $\mathrm{O}-\mathrm{H}$ proton of phenol is shown as a singlet at around $\delta$ $9.8 \mathrm{ppm}$. In the case of ${ }^{13} \mathrm{C} \mathrm{NMR}$ spectroscopy $\mathrm{CH}=\mathrm{N}$ carbon came as a sharp peak at $\delta 145-150 \mathrm{ppm}$. In the case of the metal complexes the above given IR data shows the shift towards the lower frequency of the azomethine group in the metal complex compared to the free imine.

The spectroscopic lines also decreased in the case of the metal complex compared to that of the free imine because of the increased symmetry in the complexes. So the spectral data clearly shows the formation of the imine and their corresponding complexes.

\subsection{Linear Optical, Thermal Stability and Solubility Studies of Complexes}

From the electronic absorption spectra (Figure 1), it can be observed that there is no absorption band in the visible region.

After the formation of the metal complex, a new band appear around $390 \mathrm{~nm}-420 \mathrm{~nm}$ which is due to the ligand to metal charge transfer (LMCT) of the resultant complex. The new peak is mainly attributed to the formation of co-ordination complex of Schiff base with $\mathrm{Cu}^{2+}, \mathrm{Zn}^{2+}, \mathrm{Mn}^{2+}$ and $\mathrm{Ni}^{2+}$ ion. The intensity of LMCT band was maximum for Nickel, followed by Zinc, Copper and Manganese in order.

The metal complexes synthesized were thermally stable above 360 degrees. They are completely soluble in polar solvents like methanol, ethanol, DMSO, DMF etc making the processability of the materials into thin films or devices easy.

\subsection{Non Linear Optical Studies}

The third order NLO studies were carried out for all the four compounds synthesized. All compounds showed the expected non linearity except the metal complex 1c. The non linear absorption and refraction are expressed by the equations

$$
\begin{aligned}
& \alpha(I)=\alpha+\beta I \\
& n(I)=n+n_{2} I
\end{aligned}
$$

where $\alpha$ is the linear absorption coefficient, $\mathrm{n}$ is the linear refractive index, $\beta$ is the nonlinear absorption coefficient and $\mathrm{n}_{2}$ is the non linear refractive index. According to the theory of two photon absorption process, the change in intensity of the laser beam in the unit propagation length is expressed as $\mathrm{d} I / \mathrm{d} z+\alpha I+\alpha \beta I^{2}=0$, where $\alpha$ is the attenuation coefficient caused by linear absorption and scattering, $\beta$ is the attenuation coefficient caused by non linear absorption. The non-linear two photon absorption coefficient value $(\beta)$ is obtained from the Z-scan curves. Figure 2(a) represents normalized pure non linear re-

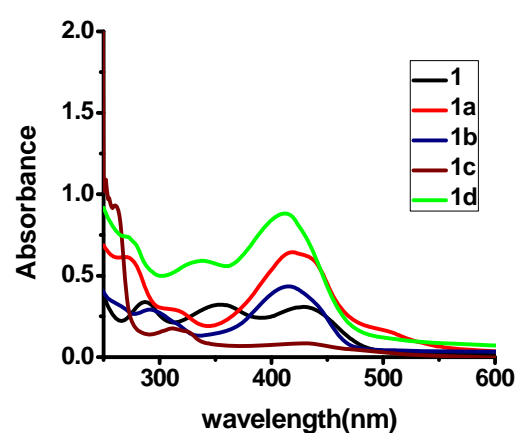

Figure 1. UV spectra of compounds $(1,1 a, 1 b, 1 c \& 1 d)$ synthesized. 
fraction curve which provides the non linear refractive index obtained by Z-scan measurements for 1a in the concentration of $1 \times 10^{-3}$ moles per litre. The measurement of transmittance without aperture enables the separation of non-linear refraction from the non linear absorption by dividing the closed aperture data by open one. The open aperture curve indicates the occurrence of non linear absorption. The graph in Figure 2(a) shows a peak followed by a valley which is a signature of negative optical nonlinearity for the samples synthesized.

This graph also represents pure refractive nonlinearity of the samples. From the graph, it is seen that the difference between the peak and the valley of the transmission curve is $\Delta T_{P V}=1.5$. Similar curves are obtained for $\mathbf{1 a}$, $\mathbf{1 b}, \mathbf{1 c}$ and $\mathbf{1 d}$ in concentration of $1 \times 10^{-3} \mathrm{M}$ as shown in Figures 2(b)-(e).

Large optical non-linearity in materials is commonly associated with resonant transitions which may be single or multi-photon nature. But for $532 \mathrm{~nm}$ outside resonant absorption, the non-linear two photon absorption coefficient value $(\beta)$ is obtained by fitting our measured transmittance values to the expression

$$
T(z)=\operatorname{In}\left[1+q_{0}(z)\right] / q_{0}(z)
$$

where $q_{0}(z)=\beta I_{0} L_{\text {eff }} /\left(1+z^{2} / z_{0}^{2}\right)$.

When there is no linear absorption, the linear absorption coefficient $\alpha \rightarrow 0, L_{\text {eff }}=L$, the thickness of the nonlinear medium. The calculated values of $\beta$ for $\mathbf{1 a}$ at 1 $\times 10^{-3} \mathrm{M}$ per liter concentration is $0.2911 \mathrm{~cm} / \mathrm{GW}$ and of non linear refractive index $\eta_{2}$ is $-0.4028 \times 10^{-11}$ esu.

Accordingly the real and imaginary parts, $\operatorname{Re} \chi^{(3)}$ and $\operatorname{Im} \chi^{(3)}$ are obtained by using $\operatorname{Re} \chi^{(3)}=2 n_{0}^{2} \varepsilon_{0} c n_{2}$ and $\operatorname{Im} \chi^{(3)}=\left(n_{0}^{2} \varepsilon_{0} c \lambda / 2 \pi\right) \beta$ and found to be $-0.4315 \times$ $10^{-13}$ esu and $0.0448 \times 10^{-13}$ esu respectively (Table 1). Same procedure was repeated to calculate the nonlinear parameters of $\mathbf{1 a}, \mathbf{1 b}$ and $\mathbf{1 d}$ in concentration $1 \times 10^{-3} \mathrm{M}$. The values obtained are given in Table 1 and are obtained by repeating $Z$-scan on each sample and the values are found to be consistent in all the trials. It should be pointed out that the Z-scan method is very sensitive as a way to locate the focus of the laser beam. Any uncertainty in the location may lead to the change in $\Delta T_{p-v}$ and the distortions appear on Z-scan signals. To compensate, the third-order nonlinearities are often associated with large error bars.

From the study it is clear that the ligand $\mathbf{1}$, metal complex 1a, 1b and 1d show good non linear optical properties. Among the metal complexes, manganese complex (1c) did not show any nonlinear optical properties. This is due to the $d^{5}$ configuration of $\mathrm{Mn}^{2+}$ ions. This halffilled orbital which is more stable configuration is responsible for the poor electron transfer between metal and the ligand system and results in poor nonlinear opti-

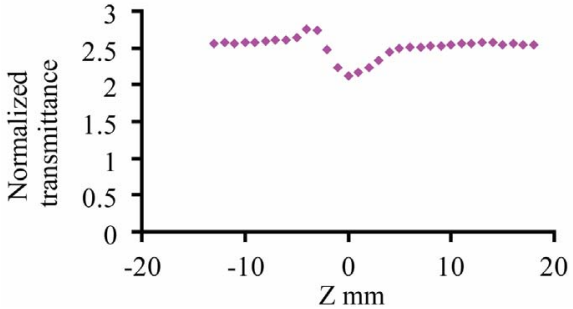

(a)

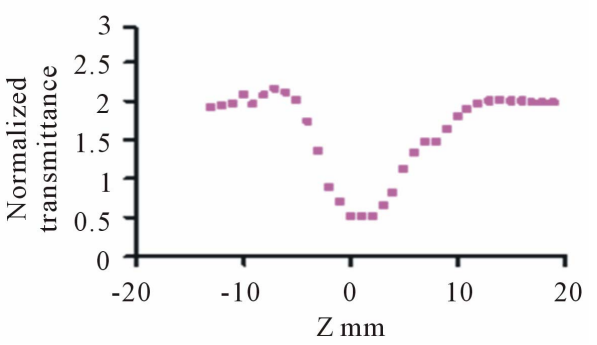

(b)

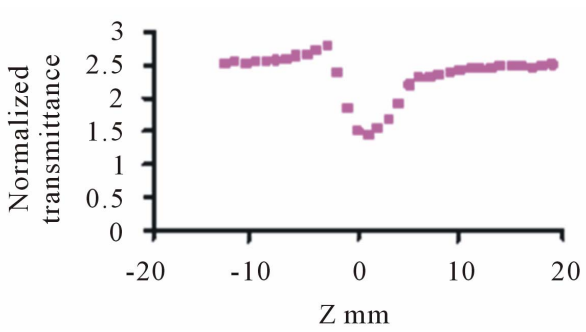

(c)

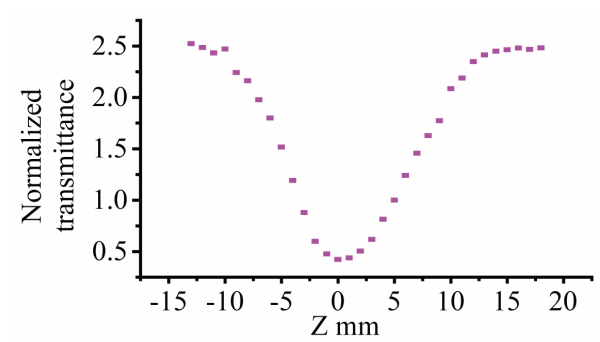

(d)

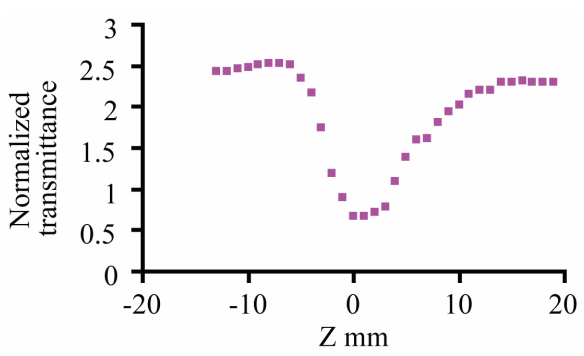

(e)

Figure 2. (a) Pure normalised Z-scan curve of 1; (b) Pure normalized $Z$ scan curve of 1a; (2c) Pure normalized $Z$ scan curve of 1b; (2d) Pure normalized Z-scan curve of 1c; (2e) Pure normalized Z-scan curve of $1 \mathrm{~d}$. 
Table 1. Third order nonlinear optical parameters of Schiff base and their metal complexes.

\begin{tabular}{ccccc}
\hline Compound & $\eta_{2}\left(\times \mathbf{1 0}^{-11} \mathbf{e s u}\right)$ & $\boldsymbol{\beta}(\mathbf{c m} / \mathbf{G w})$ & $\mathbf{R e} \chi^{3}\left(\times \mathbf{1 0}^{-13} \mathbf{e s u}\right)$ & $\mathbf{I m}^{3}\left(\times \mathbf{1 0}^{-13} \mathbf{e s u}\right)$ \\
\hline $\mathbf{1}$ & -0.9591 & 2.2294 & -1.0273 & 0.3426 \\
1a & -0.4028 & 0.2911 & -0.4315 & 0.0448 \\
1b & -1.3374 & 3.4437 & -1.4326 & 0.5293 \\
1d & -0.9247 & 1.7910 & -0.9905 & 0.2753 \\
\hline
\end{tabular}

cal properties. In the case of the other metals $\mathrm{Cu}^{2+}, \mathrm{Zn}^{2+}$ and $\mathrm{Ni}^{2+}$ the electron transfer between metal and ligand system are more feasible which results in better nonlinear optical properties.

Copper and nickel complexes were found to have better nonlinear optical properties compared to zinc complex. The transition metals especially first row transition metal ions are well known for their ability to form wide range of coordination complexes in which octahedral, tetrahedral, and square planar geometries predominate. Copper (II) is a typical transition metal ion to form complexes, but less typical in its reluctance to take up a regular octahedral (or) tetrahedral geometry. The magnitude of the splitting of the electronic energy levels in copper (II) complexes tend to be larger than other first row transition metals due to the presence of large JahnTeller distortion. Hence the electrons in the copper complexes can be easily transferred to the ligand system and vice versa. Nickel (II) complexes has $d^{8}$ electronic configuration, which is very susceptible for electronic transfer compared to zinc complex. Although zinc(II) ion is having much chemical behavior in common with copper (II) ion, due to the fully filled electronic configuration $[\mathrm{Ar}] 3 \mathrm{~d}^{10}$, the electron transfer is not feasible compared to copper and nickel complexes.

\section{Conclusion}

The third-order nonlinear parameters of Schiff base and their metal complexes have been investigated by Z-scan technique. The molecules show negative nonlinearity. The third order nonlinearity arises due to the strong delocalization of the $\pi$-electrons and the electron transfer between ligand and metal. The four molecules studied in this investigation are of the donor-acceptor type. Among the samples $(\mathbf{1}, \mathbf{1 a - d})$ examined for third-order NLO properties, manganese complex (1c) did not show NLO properties. Although the length of delocalization of electrons is the same for all metal complexes, the complexes differ in the electronic transfer taking place between metal and ligand. Hence they show different nonlinear response. Similarly the dimensionality and conformation of the metal complexes are also responsible for the difference in NLO properties.

\section{REFERENCES}

[1] D. R. Kanis, M. A. Ratner and T. J. Marks, "Design Synthesis and Properties of Molecule Based Assemblies with Large Second Order Optical Nonlinearities," Chemical Reviews, Vol. 94, No. 1, 1994, pp. 195-242. doi: $10.1021 / \mathrm{cr} 00025 \mathrm{a} 007$

[2] H. Ma, A. K. Jen and L. R. Dalton, "Polymer Based Optical Waveguide Materials Processing and Devices," $A d$ vanced Materials, Vol. 14, No. 19, 2002, pp. 1339-1365. doi:10.1002/1521-4095(20021002)14:19<1339::AID-AD MA1339>3.0.CO;2-O

[3] H. Kuhn and J. Robillard, "Nonlinear Optical Materials," CRC Press, Boca Raton, 1992.

[4] P. N. Prasad and D. J. Williams, "Introduction to Nonlinear Optical Effects in Organic Molecules and Polymers," Wiley, New York, 1991.

[5] D. S. Chemla and J. Zyss, "Nonlinear Optical Properties of Organic Molecules and Ctystals," Academic Press, Cambridge, 1987.

[6] D. J. Williams, "Nonlinear Optical Properties of Organic and Polymeric Materials," American Chemical Society, Washington DC, 1983.

[7] Ch. Bosshard, R. Spreitier, P. Gunter, R. R. Tykwinski, M. Schreiber and F. Diederich, "Structure-Property Relationship in Nonlinear Optical Tetraethynylethenes," $A d$ vanced Materials, Vol. 8, No. 3, 1996, pp. 231-234. doi:10.1002/adma.19960080309

[8] S. R. Marder, W. E. Torruellas, M. Blanch Desce, V. Riddi and G. I. Stegeman, "Large Molecular Third Order Optical Nonlinearities in Polarized Carotenoids," Science, Vol. 276, No. 5316, 1997, pp. 1233-1236. doi:10.1126/science.276.5316.1233

[9] J. L. Bredas, C. Adant, P. Tackx and A. Persoons, “A Third Order Nonlinear Optical Response in Organic Materials: Theoretical and Experimental Aspects," Chemical Reviews, Vol. 94, No. 1, 1994, pp. 243-278. doi:10.1021/cr00025a008

[10] P. Audebert, K. Kamada, K. Matsunaga and K. Ohta, "The Third Order NLO Properties of D- $\pi-$ A Molecules with Changing a Primary Amino Group into Pyrrole," Chemical Physics Letters, Vol. 367, No. 1, 2003, pp. 62-71. doi:10.1016/S0009-2614(02)01575-0

[11] Z. M. Xue, Z. M. Tang, Y. W. Wu, J. Y. Tian, Y. P. Jiang, H. M. H. Fun and K. Usman, "A crystal Structure, Spectroscopic Studies, and Nonlinear Optical Properties of Schiff Base Metal Complexes Containing an N, S Donor Ligand," Canadian Journal of Chemistry, Vol. 82, No. 12, 
2004, pp. 1700-1706. doi:10.1139/v04-142

[12] J. Tedim, S. Patricio, R. Bessada, R. Morais, C. Sousa, M. B. Marques and C. Freire, "Third Order Nonlinear Optical Properties of DA-Salen-Type Nickel(II) and Copper(II) Complexes," European Journal of Inorganic Chemistry, Vol. 17, No. 17, 2006, pp. 3425-3433. doi:10.1002/ejic.200600017

[13] H. Hou, G. Li, Y. Song, Y. Fan, Y. Zhu and L. Zhu, "Synthesis Crystal Structures and Third Order Nonlinear Optical Properties of Two Novel Ferrocenyl Schiff Base Complexes, "European Journal of Inorganic Chemistry, Vol. 12, No. 12, 2003, pp. 2325-2332. doi:10.1002/ejic.200200616

[14] M. A. Diaz Garcia, J. A. Duro and F. Fernandez Lazaro, "Third-Order Nonlinear Optical Susceptibilities of the
Langmuir-Blodgett Films of Octa-Substituted Metallophthalocyanines," Applied Physics Letters, Vol. 69, No. 1, 1996, pp. 3-5. doi:10.1063/1.118150

[15] F. Yaoting, L. Gang, L. Zifeng, H. Hongwei and M. Hairong, "Synthesis, Structure and third Order Nonlinear Optical Properties of 1,3,5-Triazine-Based Zn(II) Three Dimensional Supramolecule," Journal of Molecular Structure, Vol. 693, No. 2, 2004, pp. 217-224. doi:10.1016/i.molstruc.2004.03.008

[16] M. Sheik-Bahae, A. A. Said, T. H. Wei, D. J. Hagan, E. W. Van Stryland, "Sensitive Measurement of Optical Nonlinearities Using a Single Beam," IEEE Journal of Quantum Electronics, Vol. 26, No. 4, 1990, pp. 760-769. doi:10.1109/3.53394 